\title{
Impact of a Counseling Supervision Course on Doctoral Students' Cognitions
}

\author{
By: L. DiAnne Borders, Leslie M. Rainey, Lori B. Crutchfield, Debra W. Martin
}

This is the pre-peer reviewed version of the following article: Borders, L. D., Rainey, L. M., Crutchfield, L. B., \& Martin, D. W. (1996). Impact of a counseling supervision course on doctoral students' cognitions. Counselor Education and Supervision, 35(3), 204-217., which has been published in final form at [Link to final article].

\begin{abstract}
:
Studies the impact of a didactic and experiential supervision course on counseling students' cognitions, including perceptions of their supervisory behaviors, self appraisals of their supervision ability, conceptualizations about a specific supervisee. Results from the Supervisory Style Inventory and the Supervisor Emphasis Rating Form-Revised.
\end{abstract}

Keywords: Counseling | Education | Supervision

\section{Article:}

Pre- and post assessment measures indicated significant Improvements in students' selfappraisals of their ability to supervise, and in the quality and the clarity of their conceptualizations about a videotaped supervision session.

Increased emphasis on the supervision process has generated greater interest in charting and fostering the development of supervisors. Particular concern has been expressed about the need to establish supervisor training programs and, concomitantly, to investigate systematically the effectiveness of these programs (Borders et al., 1991; Ellis \& Dell, 1986). Yet, although the number of doctoral courses in counseling supervision has proliferated in recent years (Borders \& Leddick, 1988), empirical studies of their impact are scant. In fact, only two published outcome studies were located.

In the first study, Stenack and Dye (1983) Investigated the impact of three supervisor roles on the verbal behaviors of supervisees. Five doctoral students received training in Bernard's (1979) discrimination model, which includes the supervisor roles of teacher, counselor, and consultant. In their first analysis, the researchers found that the students could learn and could efficiently demonstrate the three supervisor roles with different supervisees. In their second analysis, they found that the role behaviors had significant effects on behavior of supervisees. When supervisors performed in the teacher role, supervisees discussed actions and thoughts; when supervisors demonstrated the counselor role, supervisees focused on feelings; when supervisors portrayed the consultant role, supervisees focused on thoughts. Based on their results, Stenack and Dye suggested that training programs such as theirs could help supervisors learn to be flexible in their supervisory behaviors, matching these to the needs and goals of supervisees. 
In the second study of supervisor training outcomes, Borders and Fong (1994) investigated changes in cognitions of nine supervisors-in-training across a one-semester supervised practicum in supervision. None of the students had completed formal course work in counseling supervision. We studied content of thoughts about supervision issues, choice of supervision interventions, and cognitive self-appraisals of supervision ability for nine trainees in two different doctoral programs. No significant pre-post differences were found for any of the cognitive-based variables, and students' intervention choices often conflicted with those of experts. In addition, students' self-appraisals did not improve; at posttest, they still rated supervision as relatively difficult and reported somewhat negative assessments of their ability to cope with the task. Although our results indicated that the one-semester practicum did not have a substantial impact on students' supervision work, we speculated that the limited supervision experience may have been inadequate for effecting change in the cognitive-based variables.

In fact, a sequence of didactic and experiential activities is recommended in professional guidelines for training supervisors (Borders et al., 1991; Dye \& Borders, 1990). Although this recommended sequence was based on a substantial body of literature on effective counselor training programs, no direct evidence exists that such a sequence is also preferable for supervisor training programs.

Thus, as a first step toward addressing this void, we conducted an investigation of the effectiveness of an introductory supervisor training course that included both didactic instruction and a supervised practicum experience. We again focused on cognitive outcome variables, given that the "cognitive shift" (Heath \& Storm, 1983) from thinking like a counselor to thinking like a supervisor (e.g., from a focus on the client to a focus on the counselor) is seen as a crucial change for supervisors-in-training (e.g., Bernard \& Goodyear, 1992;

Borders, 1989, in press; Heath \& Storm, 1983). In this study, we focused on the impact of the course on trainees' perceptions of their supervisory behaviors, their self-appraisals of supervision ability, and their conceptualizations about supervision. Specifically, we investigated the following research questions:

1. How do supervisors-in-training describe their anticipated supervision behaviors (supervisory style and focus) with first practicum master's-level students? After training, do their anticipated supervision behaviors correspond with the behaviors they believe they actually used with the practicum students? Are the posttest self-reported behaviors in line with course content?

2. Does a supervision course that includes both didactic and experiential instruction improve the self-appraisals that supervisors-in-training have of their ability to supervise?

3. Does the supervision course also have an impact on the conceptualizations that supervisors-in-training have about a supervisee and also on their plans for a specific supervision session? 


\section{METHOD}

\section{Participants}

Eleven doctoral students (across 2 academic years) volunteered to participate in the study. They were enrolled in a counselor education program in the Southeast approved by the Council for Accreditation of Counseling and Related Educational Programs. All were enrolled in a required, 3-hour semester course In counseling supervision. The group was composed of 6 women and 5 men. Nine participants were Anglo-Americans, I was African American, and I was Hispanic American. Their ages ranged from 23 to 45 years $(M=35.91, S D=7.99)$. The 11 reported a wide range of total hours of post-master's-level counseling experience ( 0 to 144 months, $\mathrm{M}=$ 47.36, $\mathrm{SD}=57.14)$. Previous teaching experience also varied ( 0 to 90 months, $\mathrm{M}:=30.36$, $\mathrm{SD}=$ 30.41). Six reported no experience as a clinical supervisor: the other 5 had worked with one or more counseling interns at their previous work :settings.

\section{Counseling Supervision Course}

The 3-hour supervision course included both didactic and experiential components along suggested professional guidelines (cf. Borders et al., 1991; Dye \& Borders, 1990). Didactic instruction involved lectures and seminar-type discussions of relevant topics, including supervision models (particularly developmental models and Bernard's, 1979, discrimination model); interventions and techniques; and ethical, legal, relationship, and evaluation issues.

As part of this course, students were required to supervise 1 or 2 first-year master's-level students enrolled in their first counseling practicum, which involved five counseling sessions with a volunteer undergraduate client. Master's-level supervisees were assigned to their doctoral student supervisors on the basis of counseling track (e.g., school counseling or community agency counseling) and schedules. The doctoral-level supervisors met with their students for a minimum of seven supervision sessions, including one introductory meeting before counseling sessions began, one meeting to review each of the five counseling sessions, and one evaluation and closure session. Supervisors were encouraged to use a minimum of two different supervisory interventions (e.g., IPR, role play, live observation) across the sessions. All counseling sessions and supervision sessions were conducted at the in-house clinic; all sessions were videotaped. During the supervision practicum, supervisors met weekly in small peer groups (alternatively observed by the instructor) to review videotapes of their supervision sessions and seek input on their questions and plans for the next session. They also met with the instructor for at least one individual. supervision session.

\section{Dependent Variables}

Supervisors' behaviors. Two measures of supervisors' perceptions of anticipated (pretest) and actual (posttest \} in-session behaviors were used. First, students described their style as supervisors on the Supervisory Styles Inventory (SSI: Friedlander \& Ward, 1984). On the SSI, 
respondents use a 7-point Likert scale ( 1 = not at all,' 7 = very) to indicate the degree. to which 33 items (adjectives) describe their in-session behaviors (7 are filler items). Subscales represent three dimensions of a supervisor's s type: attractive (collegial), inter-personally sensitive (relationship- and process-oriented), and task-oriented (practical, didactic), which correspond with three roles of the supervisor: consultant:, counselor, and teacher, respectively. Ratings on subscale items are averaged to determine each subscale score. in a series of studies, Friedlander and Ward demonstrated high reliability and validity for the instrument, including factor analyses that supported the three subscales, and significant differences in styles by supervisors' counseling orientation and by experience level of supervisees. Test-retest reliability coefficients ranged from .78 to .94 for the subscales, with .92 for the total score; coefficient alphas ranged from .70 to .93 for the subscales and .82 to .91 for the total score.

As a second measure of in-session behaviors, supervisors described their supervisory focus on the Supervisor Emphasis Rating Form-Revised (SERF-R; Lanning, 1986; Lanning \& Freeman, 1994). The SERF-R, based in part on Bernard's (1979) discrimination model, consists of 15 sets of four items; Items within each set have equal rank and all are desirable and positive supervision behaviors. One item in each set represent, s one of the four possible supervision emphases: professional behavior skills (e.g., ethical behavior), process skills (e.g., reflections, open-ended questions), personalization skills (e.g., self-awareness. of personal issues), and conceptualization skills (e.g., case conceptualization). Respondents rank order the four items within each set to indicate which item receives the most (1) to the /east (4) emphasis. Rankings are summed for a total score for each emphasis subscale so that lower scores indicate a higher ranking or emphasis for a particular subscale. Lanning and Freeman (1994) reported internal consistency coefficients for the subscales, with Cronbach's alphas ranging from .67 to .80, and split-half correlations ranging from .60 to .72, all within an acceptable range for research purposes. In addition, Lanning and Freeman stated that because the SERF-R items are identical to those on the original Likert-style instrument (Lanning, 1986), validity information available for the first version Is applicable to the revised form.

Cognitive appraisal Students indicated self-appraisals of their ability to supervise on the Stress Appraisal Scale (SAS; Carpenter \& Suhr, 1988). The SAS consists of 36 items covering six domains related to the stress appraisal process for any performance activity. Instruments are tailored for the specific activity under study (i.e., "Answer the following questions according to how you are feeling about doing supervision"). Students' responses on a 4-point Likert scale (1 = very untrue of me to 4 = very. the of me) are summed to obtain three subscale (factor) scales, with a possible range of scores of 12 to 48 on each subscale. Internal consistency estimates (coefficient alphas) for the six domains ranged from. 77 to.90 (Carpenter \& Suhr, 1988). Carpenter and Suhr also reported supportive concurrent validity data. In addition, a principal axis factor analysis yielded three factors or subscales. In line with Borders and Fong (1994), we elected to use the two factors with higher validity: difficulty (demands of the task and 
perceptions of one's stress level) and secondary or self-appraisal (skills and resources for coping, and prediction of success).

Supervisors conceptualizations. To assess the quality of supervisors' conceptualizations of a supervisee and their plans for an upcoming supervision session with that supervisee, students completed the Supervision Assessment Questionnaire (SAQ). The SAQ is an adaptation of the Clinical Assessment Questionnaire (CAQ; Holloway, 1979; Holloway \& Wolleat, 1980), a measure of counselors' clinical hypothesis formation. The CAQ was designed to apply information processing constructs to clinical hypothesis formation, and was based on the belief that "knowing the content of a clinic Jan's judgments is not enough; the manner in which the clinician arrives at his/her conclusions is also essential" (Holloway, 1979, p. 76). Three information processing constructs were considered in construction of the CAQ: (a) content of the counselor's decisions about the client (e.g., number of information elements and time frames used), (b) counselor's use of information in making those decisions (e.g., conceptual vs. objective information, internal vs. external frame of reference), and (c) counselors' search for additional information about the client (e.g., number of divergent questions asked) (Holloway, 1979). Scores on the CAQ have a positive direct relationship with conceptual level (Holloway, 1979; Holloway \& Wolleat, 1980). Achieving higher conceptual levels is a goal in supervising counselor trainees (Blocher, 1983; Loganbill, Hardy, \& Delworth, 1982; Stoltenberg, 1981) and, by extrapolation, a goal of supervisor training (Borders, in press; Stoltenberg \& Delworth, 1987)

Similar to the CAQ, the SAQ consists of five written tasks that direct respondents toward stating and supporting two hypotheses about a supervisee. Following procedures described by Holloway (1979) and Holloway and Wolleat (1980), students viewed a 15-minute videotaped segment of an actual counseling session between a 42-year-old White female counselor and her 34-year-old White female client. The segment was focused on the client's goal of a career change and her marital problems. The client expressed a range of emotions and intensity; the counselor exhibited both positive (e.g., summary statements) and negative (e.g., closed questions) counseling responses. After viewing the videotaped segment, students completed the specified tasks, including the following: "List the possible points or issues you could cover in the supervision session with this supervisee," "Choose the two points you think are most important," and "Discuss what evidence helped you form your perceptions."

Following CAQ scoring instructions, SAQ protocols were rated for the absence or the presence of six categories of information: (a) elements considered in understanding the supervisee, (b) time frames used in understanding the supervisee, (c) categories of information used to support conclusions, (d) instances used to support conclusions, (e) categories of information sought, and (f) number of divergent questions asked. As appropriate, categories were added to address additional supervision elements (e.g., supervisees "background in counseling" added to categories of information sought). For Categories a, b, c, and e, a score of 0 was as,signed if an element within that category was not present, and a score of 1 was assigned if the element was present. For Categories d and f frequency counts were made of the number of instances and the 
number of divergent questions, respectively. A total score was derived for each category. In addition, hypotheses and their substantiations were rated for overall quality and clarity ( 1 = poor, 2 = neutral $3=$ good).

Given that students were being introduced to a variety of supervision interventions during the course, we added one additional task to the SAQ. Students were asked to indicate their plans for the supervision session with the videotaped supervisee. The number of interventions cited in the plans were counted and were also classified in terms of the type of intervention selected (e.g., IPR, live observation).

Three trained raters (first through third authors) independently rated all the SAQ protocols (in a blind, random order) over a 2-month period. Interrater agreement was calculated by dividing the number of agreements by the number of agreements and disagreements. Interrater agreement was low, ranging from 36\% (Category d) to 71\% (Category b) for Individual SAQ categories, and with an overall agreement of 58\% for Categories a through $\mathrm{f}$. A similar interrater agreement (54\%) resulted for the additional, exploratory tasks created for this study. Given the difficulty of making these subjective ratings (see also Holloway, 1979), consensus discussions determined a final rating for each category and participant, and, following Holloway's (1979) suggestion, overall ratings for quality and clarity were averaged across the three Judges.

\section{Procedure}

Supervisors completed the SSI, SERF, SAS, and SAQ (in that order) during class periods of the first and 15th weeks of the course. Informed consent and a demographic form also were completed during the first week.

\section{Data Analyses}

Pretest and posttest means and standard deviations were calculated for each of the dependent variables. Tests for significant differences (i.e., paired t tests and tests for binomial proportion) between the pretest and posttest scores were calculated. In an effort to balance controls for Type I and Type II errors, a. 10 family wise error rate was used for each set of tests conducted.

\section{RESULTS}

Means and standard deviations are reported in Table 1. These data and significance test results are summarized in the following section.

On the SSI (familywise error rate of .0 .33 required), three paired t tests indicated no significant differences between pre-post sub-scale means: attractive, $\mathrm{t}(10)=.16, \mathrm{p}=.88$; interpersonally sensitive, $\mathrm{t}(10)=.70, \mathrm{p}=.50$; and task-oriented, $\mathrm{t}(10)=.69, \mathrm{p}=.50$.

On the SERF (familywise error rate of .025 required), there was a significant pre-post increase in the emphasis on conceptualization skills, $\mathrm{t}(10)=-2.95, \mathrm{p}=.015$. There were no significant 
differences for the three other subscale scores: professional behaviors, $t(10)=2.42$, Iv $=.04$; process skills, $\mathrm{t}(10)=.22, \mathrm{p}=.83$; and personalization skills, $\mathrm{t}(10)=-.38, \mathrm{p}=.72$.

On the SAS (familywise error rate of .05 required), there were significant differences in pre-post means both for the difficulty subscale, $\mathrm{t}(10)=-5.94, \mathrm{p}<.0001$; and for the self-appraisal subscale, $\mathrm{t}(10)=-14.26, \mathrm{p}<.0001$. After the course, students rated supervision as less difficult and they rated. themselves as better able to cope with the task.

For the SAQ scores, tests for binomial proportions were performed to Identify any changes In conceptualizations about the counselor or any changes In supervision plans. This particular test was necessary because of the small sample size and the restricted range of scores. The binomial test was used to determine whether the proportion of students whose conceptualization scores changed in the hypothesized direction (i.e., increased) exceeded the proportion expected by chance. In this instance, we hypothesized that one third of the responses would decrease, one third would stay the same, and one third would increase. Thus, based on a sample size of 11 and a .05 significance level, at least 7 students' responses had to Increase In order to reach significance.

Based on the binomial tests, there were no significant changes th the number of elements, time frames, categories of support, or instances of support that students cited In their SAQ protocols. For the Information Sought subscale, however, a significant number of students $(n=8)$ increased the total number of categories of additional information (e.g., significant others., cognitions, feelings about sell) they wanted about the client, the counselor, or both. Overall, however, therapy context (e.g., 'Is this session typical of the counselor's work with this client?") was the most frequently cited category at both testings. The only marked increase by category (vs. number of students) was for supervisee's background in counseling (increased from 4 to 8). In addition, on the Divergent Questions subscale, a significant number of students $(n=8)$ Increased the number of distinct and relevant questions asked (information sought) about the client, this counselor, or both.

For the overall ratings of SAQ protocols, judges' ratings of the overall quality and clarity of students' protocols were averaged for each pre-and posttest rating. Based on a paired t test, there was a significant difference in these ratings at pretest and posttest, $\mathrm{t}(10)=2.58, \mathrm{p}=.027$. Raters judged the quality and the clarity of the protocols to have improved significantly by the end of the supervision course.

Analysis of students plans for the supervision session indicated no significant difference in the number of interventions students planned to use in the session, $t(10)=.61, p=.55$. At both pretest and posttest administrations of the SAQ, students indicated that they would plan to use an average of about two separate interventions In a supervision session with the videotaped supervisee. At both administrations, the most frequent type of intervention cited was review of 
videotape and general discussion. There was an overall increase (from 0 to 5 ) in the number of times students said they planned to use IPR in the supervision session.

\section{DISCUSSION}

In this study, we investigated the impact of a didactic and experiential supervision course on students' cognitions, including perceptions of their supervisory behaviors, self-appraisals of their supervision ability, conceptualizations about a specific (videotaped) supervisee, and plans for a supervision session with that supervisee. Results were mixed and are summarized as follows.

First, the course apparently had no impact on students' perceptions of their supervisory style and had minimal impact on their perceptions of the focus areas they emphasized during actual supervision sessions. Supervisors-in-training indicated that they anticipated using and then actually used a combination of the consultant and counselor supervisory styles with their practicum students. Both before and after the course, they reported using these styles primarily to focus on developing skills and serf-awareness in their practicum students. There was, however, a significant increase in their focus on conceptualization skills.

These results both concur and contrast with those supervisor roles prescribed for beginning counseling students in developmental models of supervision (e.g., Loganbill et al., 1982; Stoltenberg, 1981), which were studied as part of the course. Developmental models would prescribe more of a teacher role for supervisors working with first-practicum master's-level students, although the high priority given to counseling skills would be theoretically appropriate. It seems, then, that study of the developmental models was minimally effective in helping students select appropriate supervisory styles and emphasis areas. Instead, for the most part, they maintained their pretraining preferences for style and emphases. These results seem to be in line with Holloway and Wolleat's (1981) findings that novice supervisors had individual, rather personalized styles that remained stable across those they supervised. Stenack and Dye (1983), however, found that given focused training in specific role behaviors doctoral students could vary their verbal behaviors with various supervisees. Such focused training may be necessary to change supervisors' in-session behaviors, an important area for future research.

It should be noted, however, that students' perceptions of their in-session behaviors may or may not have reflected their actual behaviors. There is reason to suspect that the two do not always match. In a case study of two experienced and two novice supervisors, for example, Borders (1991) found that the supervisors and their supervisees reported a consultant-counselor style of supervision, but a more task-oriented, teacher role was found when actual supervisor responses were analyzed.

In terms of the second cognitive variable, results indicated the supervision course had a substantial impact on students' self-appraisals of their supervision abilities. After the course, students reported a significant decrease in their stress appraisals and a rather dramatic increase in their beliefs about their ability to perform as supervisors. Of course, it cannot be said with 
certainty that the supervision course alone led to these changes, nor can it be surmised what aspect of the course (e.g., didactic, or experiential instruction, or both) contributed to the change.

These results, however, do contrast with our earlier study (Borders \& Fong, 1994, using the same cognitive appraisal measure) of students $[n=8$ ) who participated in a supervision practicum (experiential instruction) only. In terms of the difficulty subscale, the two groups of students began their training experiences with equally high ratings of the demands of the supervision task and of their stress levels about performing the task (difficulty subscale: practicum students, $\mathrm{M}=$ 34.56, SD = 6.19; didactic and experiential course students, $\mathrm{M}=35.09$, SD = 6.67). At posttest, students in the practicum still rated supervision as fairly difficult $(\mathrm{M}=31.33, \mathrm{SD}=3.77)$, whereas students in the didactic and the experiential courses reported a significant decrease in their difficulty ratings $(M=23.64, S D=5.89)$. In terms of the secondary appraisal subscale, students in the Borders and Fong (1994) study began their experiential-only training with confidence; they reported relatively better (lower) ratings of their skills and resources for coping and their predictions for success with the task $(\mathrm{M}=21.56, \mathrm{SD}=4.37)$, and reported similar (nonsignificant) posttest ratings $(M=16.78, S D=4.35)$. Students in this study began the course with relatively less self-confidence $(\mathrm{M}=32.64$, $\mathrm{SD}=4.88)$ than did their counterparts in the previous study, but at posttest they reported significantly improved appraisals of their ability to cope with the task $(\mathrm{M}=19.27, \mathrm{SD}=4.41)$.

Differences in the two groups' pre- and posttest scores are difficult to explain (e.g., personality differences? different. expectations based on the type of upcoming training experience?). One plausible explanation for the significant findings in this study (vs. nonsignificant findings in Borders \& Fong, 1994), is the more comprehensive training experience (i.e., didactic and experiential instruction) the students received. Further research is needed to investigate this hypothesis and then to determine the most effective combination of training elements and training sequence.

On the third measure of supervisory cognitions, results indicated only slight improvement in students' conceptualizations about the videotaped supervisee and their supervision plans. The overall quality and clarity of the protocols did increase significantly, and there were two indications of significant improvement in their divergent thinking, a characteristic of higher level cognitive functioning. Additional research is needed, however, to clarify the impact of supervisor training on supervisors' cognitive functioning.

There were few improvements in students' plans for a supervision session with the videotaped supervisee. The only notable change was the increase in plans to use Interpersonal Process Recall (IPR; Kagan, 1980). It seems that instruction. in this method, typically their first introduction to this approach, did influence students' decisions to choose it.

Several limitations of this study should be kept in mind when considering the results. The small sample size was problematic and also contributed to low power. A familywise error rate of .10 
was used in an effort to balance Type I and Type II errors, but the approach cannot compensate for low power. Low power is a consistent problem in supervision research (cf. Bernard \& Goodyear, 1992) because it often takes several years to generate even a moderate sample size of supervisors-in-training. Low interrater reliability for the SAQ indicates that results for this measure must be considered with great caution; it also is an indication of difficulties in making subjective ratings of cognitive variables. The analogue nature of the SAQ should also be considered, although the use of a videotaped (vs. written) segment of an actual counseling session did enhance its validity. Lack of a control group must also be noted. In addition, participants were from one counselor education program; possible pretest differences between students at various universities, as found by Borders and Fong (1994), are: a reminder that caution should be taken in generalizing results from only one program. Collaborative efforts by researchers at several supervisor training programs would help to address not only this problem, but also the low power issue. Finally, results were based on self-reported cognitions, including perceptions of in-session behavior. As Borders (1989) indicated, perceptions may not match actual behaviors.

As several of these limitations imply, supervision researchers currently are greatly hampered by the lack of outcome (and process) measures specifically based in the supervision enterprise (Bernard \& Goodyear, 1992; Borders \& Fong, 1994; Holloway, 1984). Measures, such as the SAQ, that are borrowed from counseling research often inadequately depict supervision events (Borders, 1991). Thus, it is difficult to determine whether the failure to find significant changes on some SAQ subscales were due to shortcomings of the supervision course or those of the SAQ, or both.

Giver, the Importance of the "cognitive shift" to thinking like a supervisor that needs to occur as a result of supervisor training (Bernard \& Goodyear. 1992: Borders, 1989, in press; Heath \& Storm, 1983), measures of supervisors' cognitions are especially needed. Such measures, however, are difficult to design, particularly if they are to assess cognitive changes such as attitude shifts or the development of a cognitive map for conducting supervision. Several students' comments on their posttest SAQ protocols suggested that such changes occurred for at least a few of them. One student wrote the following:

I don't see her [the counselor] as much as a "bad guy" now--not better, Just more human with her own values, background. issues, etc. As a result of supervision experience, I have become more tolerant, and see less than adequate counseling as an opportunity for development rather than as bad counseling.

Another made this statement:

I don't think my responses to the videotape were any different, but I felt like I had a framework for my assessment and plans. 
Given the complexity of the supervision process, creative and concerted efforts are needed to design reliable and valid measures of a variety of desired outcomes, including changes in the supervisor and influences on supervisees and their clients. This work is imperative if supervisors. counselor educators, professional counselors, and researchers are to determine the components of effective supervisor training programs.

TABLE 1 Pretest and Posttest Means and Standard Deviations of Student Supervisors' Cognitions

Measure Pretest Posttest

M SD $\mathrm{M} \quad \mathrm{SD}$

Supervisory Style Inventory
Attractive
$\begin{array}{llll}5.93 & 0.67 & 5.96 & 0.51\end{array}$
Interpersonally sensitive
$\begin{array}{llll}5.32 & 0.93 & 5.48 & 0.53\end{array}$
Task-oriented
$\begin{array}{llll}4.29 & 0.53 & 4.50 & 0.88\end{array}$

Supervisor Emphasis Rating Form--Revised[a]

$\begin{array}{llllll}\text { Professional behaviors } & 46.00 & 4.43 & 51.36 & 4.70 \\ & \text { Process skills } & 31.09 & 4.85 & 31.45 & 5.35 \\ \text { Personalization skills } & 32.18 & 7.57 & 31.45 & 4.74 \\ & \text { Conceptualization skills } & 40.73 & 8.42 & 35.73 & 5.44\end{array}$

Stress Appraisal Scale

Difficulty

$35.09 \quad 6.67 \quad 23.64 \quad 5.89$

Secondary appraisal

$32.64 \quad 4.88 \quad 19.27 \quad 4.41$

Supervisor Assessment Questionnaire
Elements
$\begin{array}{llll}1.91 & 0.30 & 2.09 & 0.30\end{array}$ 


$\begin{array}{lllll}\text { Time frames } & 1.73 & 0.65 & 2.18 & 0.40 \\ \text { Categories of support } & 2.73 & 0.90 & 3.09 & 1.04 \\ \text { Instances of support } & 4.18 & 1.25 & 4.27 & 1.74 \\ \text { Information sought } & 2.09 & 1.14 & 3.09 & 1.22 \\ \text { Divergent questions } & 2.82 & 1.66 & 4.73 & 1.90 \\ \text { Overall ratings of protocols } & 2.15 & 0.40 & 2.44 & 0.33 \\ \text { Types of interventions } & 2.27 & 0.79 & 2.45 & 0.52 \\ \text { Focus of sessions } & 2.00 & 0.45 & 2.00 & 0.77\end{array}$

Effectiveness ratings of supervisee $\quad \begin{array}{llll}2.77 & 0.56 & 2.73 & 0.79\end{array}$

a Due to rank ordering of responses on the SERF-R, lower scores indicate higher emphasis ratings.

\section{REFERENCES}

Bernard, J. M. (1979). Supervisor training: A discrimination model. Counselor Education and Supervision, 19, 60-68.

Bernard, J. M., \& Goodyear, R. K, (1992). Fundamentals of clinical supervision. Needham Heights, MA: Allyn \& Bacon,

Blocher, D. H. (1983). Toward a cognitive developmental approach to counseling supervision. The Counseling Psychologist, 11(1), 27-34.

Borders, L. D. \{1989). A pragmatic agenda for developmental supervision research. Counselor Education and Supervision, 29, 16-24.

Borders, L. D, (1991). Supervisors' in-session behaviors and cognitions. Counselor Education and Supervision, 31, 32-47.

Borders, L. D. \{in press). Training programs for supervisors. In A. K. Hess (Ed.), Psychotherapy supervision: Theory, research and practice (Vol. 2). New York: Wiley.

Borders, L. D., Bernard, J. M., Dye, H. A., Fong, M. L,, Henderson, P., \& Nance, D. W. (1991). Curriculum guide for training counseling supervisors: Rationale, development. and implementation. Counselor Education and Supervision, 31, 58-80.

Borders, L. D., \& Fong. M. L. (1994). Cognitions of supervisor-in-training: An exploratory study. Counselor Education and Supervision. 33, 280-293. 
Borders, L. D., \& Leddick, G. R. (1968), A nationwide survey of supervision training. Counselor Education and Supervision. 27, 271-283.

Carpenter, B. N., \& Suhr, P. (1988, August). Stress appraisal: Measurement and correlates. Paper presented at the annual meeting of the American Psychological Association, Atlanta, GA.

Dye, H. A., \& Borders, L. D. (1990). Counseling supervisors: Standards for preparation and practice. Journal of Counseling \& Development, 69, 27-32.

Ellis, M. V., \& Dell. D. M. (1986). Dimensionality of supervisor roles: Supervisors' perceptions of supervision. Journal of Counseling. Psychology, 33, 282-291.

Friedlander, M. L., \& Ward. L. G. (1984). Development and validation of the Supervisory Styles inventory. Journal of Counseling Psychology, 31, 541-557.

Heath, A. W., \& Storm, C. L. (1983). Answering the call: A manual for beginning supervisors. The Family Therapy Networker, 7(2), 36-37, 66,

Holloway, E. L. (1979). The effects of conceptual level on clinical hypothesis formation and the supervision interview (Doctoral dissertation, University of Wisconsin-Madison, 1979).

Dissertation Abstracts International 40, 3121A.

Holloway, E. L. (1954). Outcome evaluation in supervision research. The Counseling Psychologist, 12(4), 167-174.

Holloway, E. L., \& Wolleat, P. L. (1980). Relationship of counselor conceptual level to clinical hypothesis formation. Journal of Counseling Psychology, 27, 539-545.

Holloway. E. L., \& Wolleat, P. L. (1981). Style differences of beginning supervisors: An interactional analysis. Journal of Counseling Psychology, 28, 373-376.

Kagan, N. (1980). Influencing human interaction--Eighteen years with IPR. In A. K. Hess (Ed.), Psychotherapy supervision: Theory, research and practice (pp. 262283). New York: Wiley.

Lanning, W. L. (1986). Development of the Supervisor Emphasis Rating Form. Counselor Education and Supervision, 25, 191-196.

Lanning, W., \& Freeman, B. (1994). The Supervisor Emphasis Rating Form-Revised. Counselor Education and Supervision, 33, 294-304.

Loganbill, C., Hardy, E., \& Delworth, U. (1982). Supervision: A conceptual model. The Counseling Psychologist, 10(1), 3-42.

Stenack, R. J., \& Dye, H. A. (1983). Practicum supervision roles: Effects on supervisee statements. Counselor Education and Supervision, 23, 157-168. 
Stoltenberg. C, (1981). Approaching supervision from a developmental perspective: The counselor complexity model. Journal of Counseling Psychology. 28, 59-65.

Stoltenberg, C. D., \& Delworth, U. (1987). Supervising counselors and therapists: A developmental approach. San Francisco, CA: Jossey-Bass.

By L. DIANNE BORDERS, LESLIE M. RAINEY, LORI B. CRUTCHFIELD and DEBRA W. MARTIN

L. DiAnne Borders is an associate professor of counselor education, and Debra W. Martin is a doctoral student, both at the University of North Carolina at Greensboro. Leslie M. Rainey is an assistant professor of counselor education at Lenoir Rhyne College, Hickory, North Carolina.

Lori B. Crutchfield is an assistant professor of counselor education at Columbus College, Columbus, Georgia. Preliminary results of this study were presented at the annual meeting of the Southern Association of Counselor Education and Supervision, Charlotte, North Carolina, November 1994. Correspondence regarding this article should be sent to L. DiAnne Borders, 228 Curry Building, University of North Carolina at Greensboro, Greensboro, NC 27412-5001. 